\title{
Multi-component parametric inversion of galaxy kinematics and stellar populations using full spectral fitting
}

\author{
Ivan Yu. Katkov and Igor V. Chilingarian \\ Sternberg Astronomical Institute, Moscow State University, \\ Universitetskii pr. 13, Moscow, 119992 Russia \\ email IK: katkov.ivan@gmail.com; IC: chil@sai.msu.ru
}

\begin{abstract}
The stellar line-of-sight velocity distribution (LOSVD) can be strongly asymmetric in regions where the light contributions of both disc and bulge in spiral and lenticular galaxies are comparable. Existing techniques for the stellar kinematics analysis do not take into account the difference of disc and bulge stellar populations. Here we present a novel approach to the analysis of stellar kinematics and stellar populations. We use a two-component model of spectra where different stellar population components are convolved with pure Gaussian LOSVDs. For this model we present Monte-Carlo simulations demonstrating degeneracies between the parameters.
\end{abstract}

Keywords. Methods: data analysis, galaxies: kinematics and dynamics, galaxies: stellar content.

\section{Introduction}

A flat rotating stellar disc and a slowly rotating spheroidal bulge in spiral and lenticular galaxies usually possess very different stellar population properties. Consequently, the resulting stellar line-of-sight velocity distribution (LOSVD) can be strongly asymmetric in regions where the light contributions of both disc and bulge are comparable. At the first approximation, this can be accounted by the Gauss-Hermite parametrization of the LOSVD (van der Marel \& Franx, 1993), however, different absorption features (e.g. sensitive to age and metallicity) will have different effective LOSVDs. The first attempt to recover parametrically the multi-component dynamics connected to multi-component stellar populations was done by De Bryuine et al. (2004), who used stellar spectra to model different stellar populations. Here we present a more realistic approach based on the full spectral fitting, where multiple dynamical components are represented by multiple simple stellar population (SSP) models. More sophisticated stellar population models can be used instead of SSPs.

\section{Two-component parametric LOSVD recovery}

As an example, we present a two-component model having two pure Gaussian LOSVD components with different stellar populations characterised by their ages and metallicities. An optimal template is represented by the linear combination of two SSPs each convolved with its own LOSVD; hence the $\chi^{2}$ value is computed as:

$$
\chi^{2}=\sum_{N_{\lambda}} \frac{\left[F_{i}-P_{p} \cdot \sum_{j} k_{j} \cdot S\left(T_{j}, Z_{j}\right) \otimes \mathcal{L}\left(v_{j}, \sigma_{j}\right)\right]^{2}}{\delta F_{i}^{2}},
$$

where $\mathcal{L}(v, \sigma)$ - pure Gaussian LOSVD; $F_{i}$ and $\delta F_{i}$ are the observed flux and its uncertainty; $S\left(T_{j}, Z_{j}\right)$ is the flux from the $j$-th synthetic spectrum of SSP with given age $T_{j}$ 


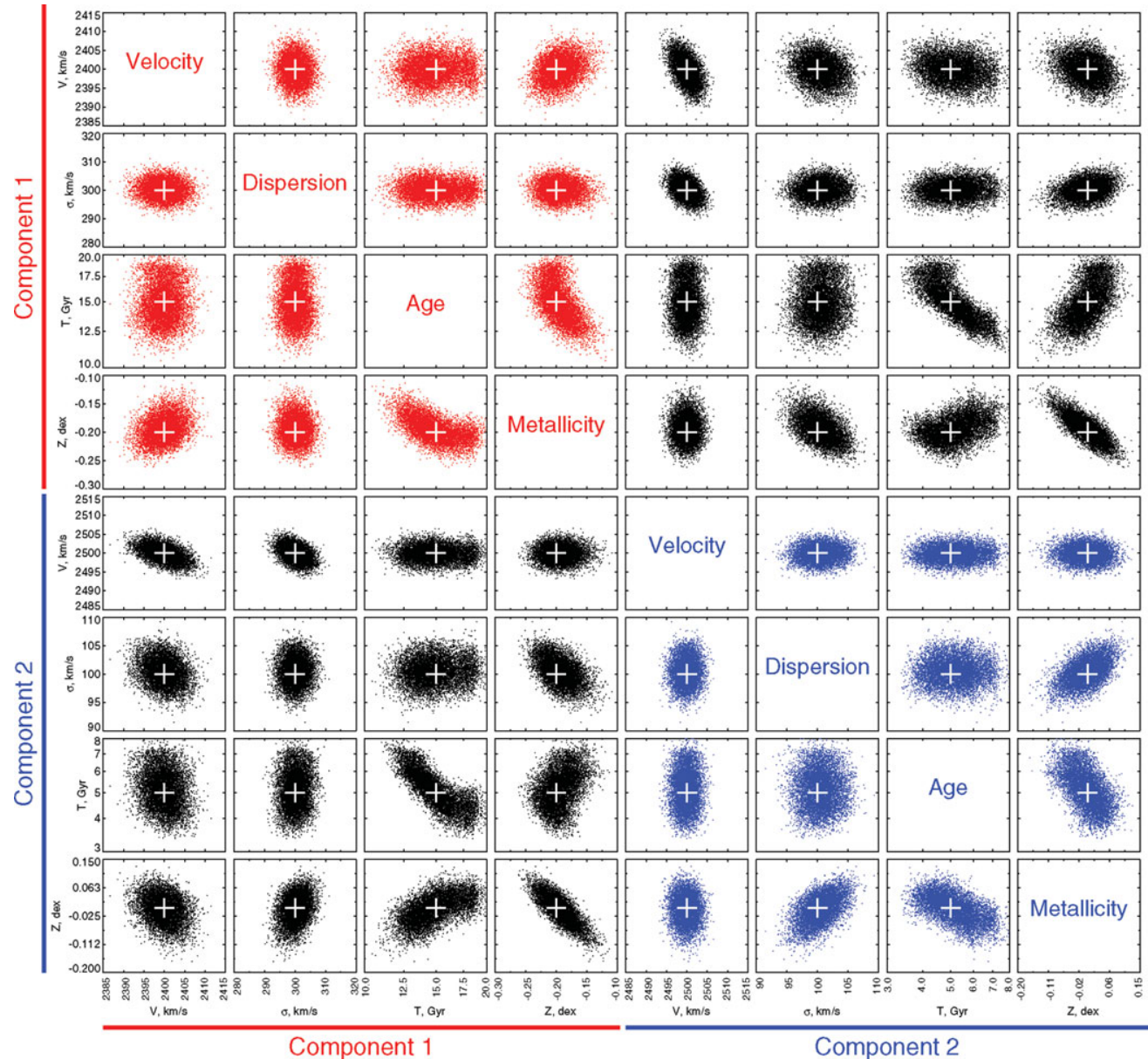

Figure 1. Covariance matrix of the model parameters. Left-top quadrant and right-bottom quadrant present a self-correlation of parameters of bulge and disc components, correspondingly. Left-bottom and right-top quadrants correspond to mutual correlation of stellar components. White crosses show the input values.

and metallicity $Z_{j} ; P_{p}$ is a multiplicative Legendre polynomial of order $p$ for correcting the continuum, determined at each step of minimization loop by solving the linear least-square problem; $k_{j}$ is the $\mathrm{j}$-th component weight (normally found by the linear minimization). The important point in this study is that we fixed the relative SSP contributions $k_{j}$ to the values derived from the photometric light profile decomposition. This approach was implemented on top of the NBURSTs full spectral fitting technique (Chilingarian et al. 2007a, Chilingarian et al. 2007b).

Our new approach was applied to the real spectra of the luminous early-type galaxy NGC 524 (see Katkov et al. 2011). It was shown that the LOSVD of NGC 524 exhibits

Table 1. Parameter of stellar components.

\begin{tabular}{lccccc}
\hline Component & $v, \mathrm{~km} / \mathrm{s}$ & $\sigma, \mathrm{km} / \mathrm{s}$ & Age, Gyr & $\mathrm{Z}$, dex & Weight $K_{j}$ \\
\hline 1 (Bulge) & 0 & 300 & 15 & -0.2 & 0.7 \\
2 (Disc) & 100 & 100 & 5 & 0.0 & 0.3 \\
\hline
\end{tabular}



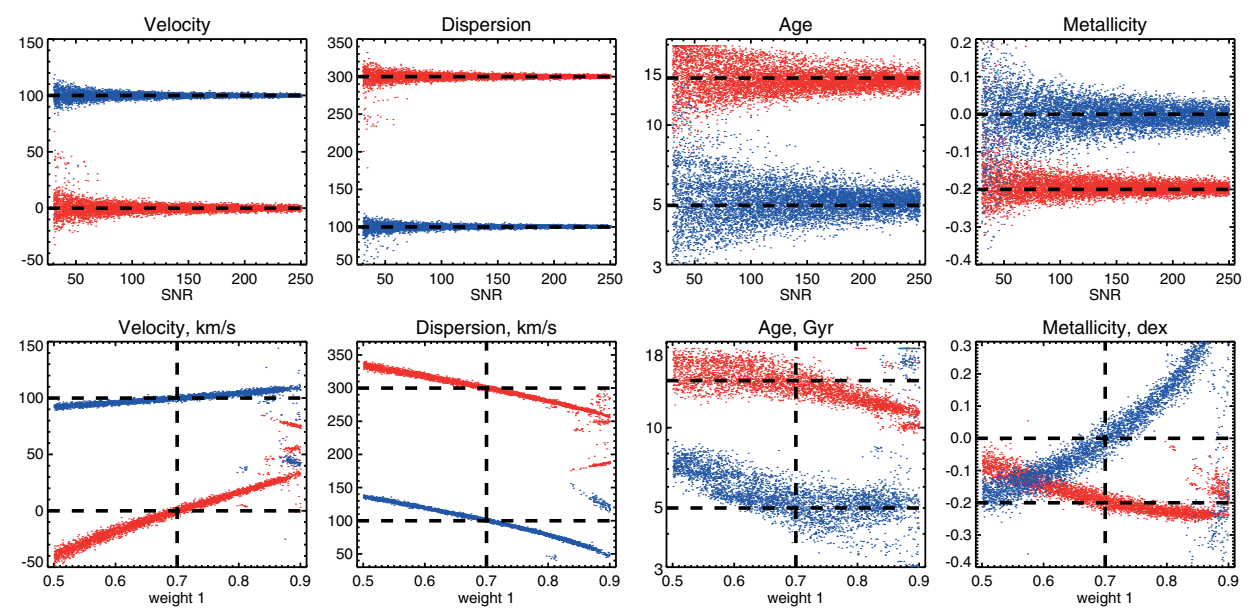

Figure 2. Top line: The dependence on signal-to-noise ratio of output parameter dispersion. Bottom line: Output parameters with varied relative contribution of components.

strong asymmetry and a stellar component of galaxy can be described as a bulge, inner disc and a conterrotating outer disc.

\section{Monte-Carlo simulation}

In order to explore the parameter space, we constructed and analysed using our technique a sample of 8000 realizations of synthetical spectra created as a linear combination of two components (see Table 1), adding random noise corresponding to the signal-noise ratio (SNR) 100. The relative contributions $k_{1,2}$ were fixed. The degeneracies between all the parameters are shown in Fig. 1 as a covariance matrix. Here and after red and blue points correspond to the bulge and disc components respectively. We can see that the most degenerate pair of parameters are $T_{1}-T_{2}$ and $Z_{1}-Z_{2}$.

In order to demonstrate how the parameters are recovered depending on the SNR we fitted a sample of 8000 models varying a SNR between 30 and 250. Fig. 2(top line) shows how the output parameter uncertainty decreases when increasing SNR. The dashed lines show the input parameters of synthetic spectrum.

We used the same approach to demonstrate the importance of knowledge of the relative contributions $k_{j}$. Fig. 2 (bottom line) presents $4000 \mathrm{MC}$ realizations where we varied the relative SSP contribution of bulge component $k_{1}$ in a range between 0.5 and 0.9 , while the real value was equal to 0.7 . SNR $=150$ was adopted in these realisations. It is obvious that even a small offset in $k_{j}$ can lead to important biases in the recovered parameters.

The authors thank the IAU for the provided financial aid and RFBR grant 10-02-00062 for covering the remaining expanses.

\section{References}

Chilingarian, I. V., Prugniel, P., Sil'chenko, O. K., \& Afanasiev, V. 2007, MNRAS, 376, 1033

Chilingarian, I. V., Prugniel, P., Sil'chenko, O. K., \& Koleva, M. 2007, In: Stellar Populations as Building Blocks of Galaxies, Proc. of the IAU Symp. no 241, eds. A. Vazdekis and R. F. Peletier, p. 175, arXiv:0709.3047

De Bryuine, V., De Rijcke, S., Dejonghe, H., \& Zeilinger, W. W. 2004, MNRAS, 349, 461

Katkov, I., Chilingarian, I., Sil'chenko, O., Zasov, A., \& Afanasiev V. 2011, arXiv:1106.2527

van der Marel, R. \& Franx, M. 1993, ApJ, 407, 525 